\title{
Association of CD117 and HLA-DR expression with shorter overall survival and/or progression-free survival in patients with multiple myeloma treated with bortezomib and thalidomide combination treatment without transplantation
}

\author{
HUAN WANG ${ }^{*}$, XIN ZHOU* ${ }^{*}$ JIAN-WEI ZHU, JIAN-NAN YE, HONG-FENG GUO and CHAO SUN \\ Department of Hematology, Affiliated Wuxi People's Hospital, Nanjing Medical University, Wuxi, Jiangsu 214023, P.R. China
}

Received November 11, 2017; Accepted June 27, 2018

DOI: $10.3892 / \mathrm{ol} .2018 .9365$

\begin{abstract}
Certain immunophenotypes in multiple myeloma (MM), including CD56 and CD117, have been reported to be associated with overall survival (OS). However, previous reports have ignored the impact of different treatment regimens and the long-term prognostic value of immunophenotyping in MM when treated with novel agents, including thalidomide and bortezomib, in the absence of transplantation for autologous stem cell transplantation and allo-hematopoietic stem cell transplantation. To further understand the long-term prognostic value of immunophenotyping in MM, when treated with bortezomib combined with thalidomide-based regimens without transplantation, 80 patients who were newly diagnosed between January 2007 and December 2015, were analyzed retrospectively. In contrast to previous studies, no significant survival time difference was observed between CD56+/CD117+ and CD56-/CD117- groups. Multivariate analysis suggested that human leukocyte antigen-antigen D-related (HLA-DR)+ was independently associated with shorter OS and progression-free survival (PFS), while CD117+ was an independent prognostic factor for decreased PFS. In addition, the myeloma prognostic index (MPI), defined by HLA-DR+, age $\geq 65$ years and international staging system stage III, was suitable for risk stratification of patients treated with novel agents for OS and PFS. The results of the current study suggested that HLA-DR+ patients had a shorter OS and PFS and CD117+ patients had shorter PFS. HLA-DR+ or CD117+ was sufficient to affect survival. Evaluating these markers may reveal valuable prognostic factors for $\mathrm{MM}$ in patients receiving bortezomib
\end{abstract}

Correspondence to: Dr Chao Sun, Department of Hematology, Affiliated Wuxi People's Hospital, Nanjing Medical University, 299 Qingyang Road, Wuxi, Jiangsu 214023, P.R. China

E-mail: 13057302309sun@163.com

${ }^{*}$ Contributed equally

Key words: multiple myeloma, bortezomib, thalidomide, human leukocyte antigen-antigen D-related, CD117 combined with thalidomide-based regimens without autologous stem cell transplantation and allo-hematopoietic stem cell transplantation). MPI may describe an accessible tool to predict the prognosis of patients with MM.

\section{Introduction}

Multiple myeloma (MM) is an aggressive hematologic malignancy, which has short overall survival time (OS) despite treatment with immunoregulatory drugs (IMiDs) and proteasome inhibitors, including bortezomib (1). It accounts for $1 \%$ of all malignant diseases and for slightly more than $10 \%$ of all hematologic cancers (2). The mean age of affected individuals is 72 years for men ( $75 \%>70$ years) and 71 years for women (79\% >70 years) (3). In addition to genetic markers, there are further prognostic factors, including lymphocyte counts and C-reactive protein (CRP) expression level, which have been evaluated in recent years $(4,5)$. Immunophenotyping, including the analysis of CD19, CD45, CD56 and CD117, is of great value to the prognosis and diagnosis of $\mathrm{MM}$ according to recent reports $(6,7)$. Pan et al (8) observed that for patients with MM, CD56+ was an independent prognostic factor for increased OS. Ceran et al (9) suggested that CD56- and CD117-groups were associated with advanced stages of MM. However, these results are based on small sample sizes (50 cases and 34 cases) $(8,9)$ and short follow-up period ( $\sim 6$ years) (8) Importantly, bias originating from different treatments was not accounted for, particularly in patients that did not receive transplantations as a result of financial status or by personal choice. Furthermore, at present, to the best of our knowledge, HLA-DR has not been studied in terms of patients with MM. The present study retrospectively analyzed clinical parameters, including immunophenotypes and prognosis, of 80 patients newly diagnosed with MM that did not receive autologous stem cell transplantation and allo-hematopoietic stem cell transplantation and was based on uniform combination treatment of bortezomib and thalidomide between January 2007 and December 2015.

\section{Patients and methods}

Patients. A total of 80 newly diagnosed patients (46 males and 34 females) with MM treated with a combination 
treatment including thalidomide between January 2007 and December 2015 in Wuxi People's Hospital (Wuxi, China) were analyzed retrospectively. Data were thought to be related to MM when they were different from our reference values. Our lab has its own reference values generated from healthy patients who were examined in our hospital and were considered as a control group Therefore, when patients with MM have data outside these levels, this was taken indicate MM. All patients had the full clinical information (age $\geq 65$ years, sex, ISS staging III, Complex Chromosome, Hypertension, Diabetes, treatment regimens and survival data) including laboratory parameters (albumin $<35 \mathrm{~g} / 1$, calcium $>2.8 \mathrm{mmol} / 1$, hemoglobin $<100 \mathrm{~g} / 1$, lymphocyte counts $>1.3 \times 10^{9} / 1$, lactate dehydrogenase $>245 \mathrm{U} / 1$, serum creatinine concentrations $>177 \mathrm{umol} / 1, \mathrm{C}$-reactive protein (CRP) expression level $>8 \mathrm{mg} / \mathrm{l}$, Flow cytometry results, survival data and immunoglobulin type of monoclonal protein) before any therapy. Concurrence of autoimmune disease, human immunodeficiency virus (HIV) and syphilis was excluded for all enrolled individuals. Karyotypes detected were based on traditional reverse-banding and/or G-banding techniques. Demographic and clinical characteristics of the patients were collected by reviewing medical charts and electronic records. Diagnostic criteria and risk stratification of the disease were based on the International Myeloma Working Group (IMWG) criteria and International Staging System (ISS) (10,11).

Flow cytometry. Immunophenotype evaluation was performed using a flow cytometer (FACS Canto; BD Biosciences, San Jose, CA, USA). Analysis were performed, using the FACS DIVA 6.1.3 software (BD Biosciences). All samples were anticoagulated with EDTA tube and examined within $6 \mathrm{~h}$. Approximately $100 \mathrm{ul}$ of anticoagulated bone marrow sample was labeled with pre-conjugated monoclonal antibodies at $25^{\circ} \mathrm{C}$ for $20 \mathrm{~min}$ in the dark. Following incubation, red blood cells were lysed and washed in PBS three times. These regents were supplied by BD Biosciences. All erythrocyte-lysed bone marrow (BM) samples obtained prior to treatments were stained using the following 3-color surface combinations (They were not as a kit and they were combined by our laboratory supplied by BD Biosciences), with fluorescein (FITC), phycoerythrin (PE) and peridinin chlorophyll protein complex (PerCP): CD2/CD19/CD45, CD3/CD56/CD45, CD4/CD8/CD45, CD5/CD7/CD45, human leukocyte antigen-antigen D-related (HLA-DR)/CD10/CD45, CD20/CD117/CD45, CD22/CD14/CD45, CD3/CD13/CD45, CD20/CD33/CD45 and CD38/CD34/CD45. Positivity was defined as $\geq 20 \%$ antibody expression and negativity as $<20 \%$ expression. IgG1 was stained with PE or with FITC as isotope/negative control at $25^{\circ} \mathrm{C}$ for $15 \mathrm{~min}$ in the dark. Each patient sample was divided into ten tubes to detect the various immunophenotype combinations listed above. All positive results from the same patient were recorded. MM are thought to be CD38+. All samples were tested CD38+ (>20\% expression). Samples containing myeloid antigens, including CD13 and CD33 in $\geq 1$ tube, were recruited as myeloid antigens-positive. All agents were provided by BD Biosciences. Cat. nos. IgG1, 349041; CD45, 652803; HLA-DR, 347363; CD10, 340921; CD20, 652808; CD117, 340129; CD22, 347573; CD4/CD8, 340039; CD14, 557742; CD38, 340909; CD34, 652802; CD13,
652820; CD33, 347787; CD2, 555327; CD19, 557791; CD3, 557052; CD56, 556647; CD5, 561896; CD7, 555360. (Dilution with aqueous buffered solution containing $\leq 0.09 \%$ sodium azide.)

Therapeutic regiments. All patients received thalidomide (100-125 mg/day) daily as basic treatment. Bortezomib and dexamethasone were additionally administered as induction and maintenance therapy. Treatment included a 3-week cycle of $1.3 \mathrm{mg} / \mathrm{m}^{2}$ bortezomib on days $1,4,8$ and 11 , with $20 \mathrm{mg}$ dexamethasone on days $1,2,4,5,8,9$ and 11,12 (12,13). Bortezomib and dexamethasone were administered at the same time. All patients received 1-3 cycles of induction therapy followed by $>2$ cycles of maintenance therapy according to the OS (Table I). Hematopoietic stem cell transplantation was not performed as a result of financial status or by personal choice; however, some patients did not qualified for transplantation.

Efficacy and follow-up. Efficacy was evaluated using the response criteria for MM provided by the IMWG (14). OS was measured from the date of diagnosis to the date of death or the last follow-up in September 2017. Progression free survival (PFS) was calculated from the date of diagnosis to the first date of confirmed progression or death by any cause.

Statistical analysis. Differences among groups were analyzed by Kruskal-Wallis and Mann-Whitney U test for continuous parameters, and by Chi-square tests for categorical data. OS and PFS were analyzed using Kaplan-Meier tests. Differences between survival curves were assessed for statistical significance using the two-tailed log-rank test. Potential risk factors for OS and PFS were evaluated in univariate and multivariate analyses using the Cox proportional hazards regression model. Hazard ratios (HRs) were estimated with $95 \%$ confidence intervals for the survival analysis. A prognostic index was designed using the variables that were the most significant prognostic factors for the multivariate analysis. The myeloma prognostic index (MPI) attributed 1 point for each unfavorable factor. Risk categories were stratified into low (score, 0 ), intermediate (score, 1) and high (score, 2-3). All data were analyzed using SSPS (v21.0, IBM Corp., Armonk, NY, USA) and $\mathrm{P}$-values are two-sided. $\mathrm{P}<0.05$ was considered to indicate a statistically significant difference.

\section{Results}

Baseline characteristics of patients with MM. Baseline characteristics of the 80 patients with MM, including age and ISS staging are presented in Table II. All patients were positive for CD38 and further immunophenotypes are presented in Figs. 1 and 2. All results were based on the flow cytometry measurements. Data from different patients is presented in Fig. 2A-D and data in Fig. 2A and $\mathrm{E}$ were obtained from the same patient. IgG1 was stained with PE (Fig. 2A-D) or with FITC (Fig. 2E) as isotope/negative control.

Univariate and multivariate analysis for $O S$ and PFS. According to the univariate analysis, age $\geq 65$ years, ISS stage III, CRP levels $>8 \mathrm{mg} / \mathrm{l}$, lactate dehydrogenase activity $>245$ U/1, $\geq 3$ abnormal (complex) chromosomes and HLA-DR+ 
Table I. Total number of cycles that patients with multiple myeloma and various immunophenotypes were administered a combination treatment.

\begin{tabular}{lcc}
\hline Groups & $\begin{array}{c}\text { Treatment cycles } \\
\text { [range (median) }\end{array}$ & P-value \\
\hline CD56+/CD117+ & $3-8(5.5)$ & 0.873 \\
CD56+/CD117- & $1-8(6.5)$ & \\
or CD56-/CD117+ & & \\
CD56-/CD117- & $4-8(6.5)$ & \\
HLA-DR+ & $1-8(4.5)$ & 0.753 \\
HLA-DR- & $2-8(5.5)$ & \\
CD56+/HLA-DR+ & $4-8(6.5)$ & 0.456 \\
CD56+/HLA-DR- & $1-8(5.5)$ & \\
CD117+/HLA-DR+ & $2-4(3)$ & 0.136 \\
CD117+/HLA-DR- & $4-8(4)$ & \\
CD13+ or CD33+/HLA-DR+ & $2-4(3)$ & 0.071 \\
CD13+ or CD33+/HLA-DR- & $4-9(5)$ & \\
MPI score 0 & $4-10(5)$ & 0.514 \\
MPI score 1 & $1-9(4.5)$ & \\
MPI score 2-3 & $1-8(5)$ & \\
\hline
\end{tabular}

Combination treatment described a 3 -week cycle of $1.3 \mathrm{mg} / \mathrm{m}^{2}$ bortezomib on days $1,4,8$ and 11 , with $20 \mathrm{mg}$ dexamethasone on days $1,2,4,5,8,9$ and 11,12 and 100-125 mg/day thalidomide. Bortezomib and dexamethasone were administered at the same time. MPI, myeloma prognostic index; HLA-DR, human leukocyte antigen-antigen D-related.

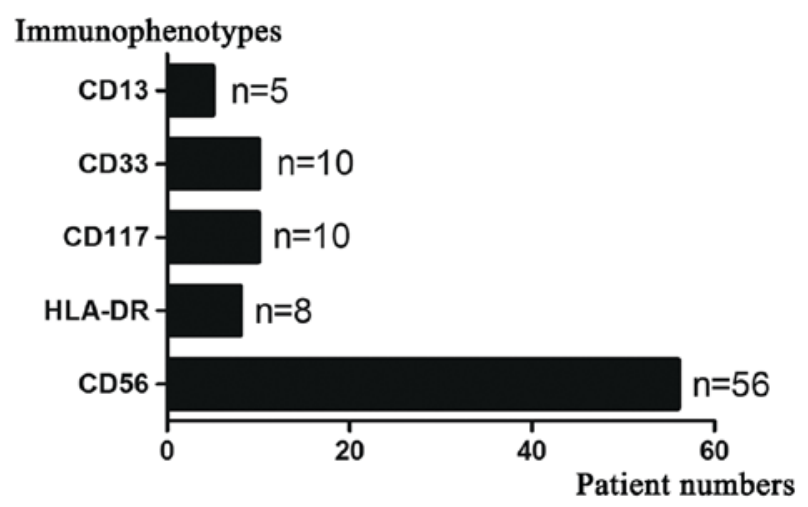

Figure 1. Number of patients with positive CD13, CD33, CD117, HLA-DR and CD56 expression. HLA-DR, human leukocyte antigen-antigen D-related.

were associated with decreased OS, while CRP levels $>8 \mathrm{mg} / \mathrm{l}$, complex chromosomes, diabetes, CD117+ and HLA-DR+ were associated with decreased PFS (Table III). In the multivariate analysis, HLA-DR+, ISS stage III and age $\geq 65$ years represented independent predictive factors for decreased OS and HLA-DR+, CD117+ and diabetes were independently correlated with decreased PFS based on the Cox proportional hazard model (Table IV).

Comparison of the expression of immunophenotypes based on 80 patients with MM. According to the expression of CD56 and CD117, patients were divided into three groups:
Table II. Baseline characteristics for patients newly diagnosed with multiple myeloma.

\begin{tabular}{|c|c|}
\hline Characteristic & Number or median (range) \\
\hline Age, years & $62(24-80)$ \\
\hline \multicolumn{2}{|l|}{ Sex } \\
\hline Male & 46 \\
\hline Female & 34 \\
\hline \multicolumn{2}{|l|}{ ISS stage } \\
\hline I-II & 40 \\
\hline III & 40 \\
\hline Hemoglobin, g/l & $88(45-148)$ \\
\hline Lymphocyte count, $x 10^{9} / 1$ & $1.20(0.16-3.95)$ \\
\hline C-reactive protein, mg/l & $5(1-160)$ \\
\hline Lactate dehydrogenase, U/1 & $128.50(50-1,221)$ \\
\hline Creatinine, $\mu \mathrm{mol} / 1$ & $97.90(30.30-760.90)$ \\
\hline $\mathrm{Ca}^{2+}, \mathrm{mmol} / \mathrm{l}$ & $2.20(1.38-5.35)$ \\
\hline Albumin, g/l & $28.20(11.70-44.10)$ \\
\hline$\beta-2$ microglobulin, $\mathrm{mg} / \mathrm{l}$ & $5.45(1.50-81.88)$ \\
\hline \multicolumn{2}{|l|}{ Isotope } \\
\hline $\operatorname{Ig} \mathrm{A}$ & 24 \\
\hline $\operatorname{IgG}$ & 38 \\
\hline Non-secrete isotype & 2 \\
\hline Light chain only & 13 \\
\hline Others & 3 \\
\hline Plasma cell in bone marrow, $\%$ & $27.75(10.50-95)$ \\
\hline \multicolumn{2}{|l|}{ Chromosome } \\
\hline$<3$ abnormal & 66 \\
\hline$\geq 3$ abnormal & 14 \\
\hline Hypertension & $30 / 50$ \\
\hline Yes & 30 \\
\hline No & 50 \\
\hline \multicolumn{2}{|l|}{ Diabetes } \\
\hline Yes & 17 \\
\hline No & 63 \\
\hline
\end{tabular}

ISS, international staging system.

CD56+/CD117+, CD56-/CD117- and CD56+/CD117- or CD56-/CD117+. No significant differences were observed. (Table V). HLA-DR+ was closely associated with complex chromosomes (Table VI) however there were no significant differences among other factors (Table VI). OS and PFS exhibited no significant differences among these groups. (CD56+/CD117+, CD56-/CD117- and CD56+/CD117- or CD56-/CD117+) (Fig. 3A and B).

Analysis of OS and PFS in different immunophenotypes. As presented in Fig. 4, the HLA-DR+ immunophenotype exhibited an adverse prognosis compared with the HLA-DRimmunophenotype for OS (12 vs. 48 months; $\mathrm{P}=0.002)$ and PFS (9 vs. 33 months; $\mathrm{P}=0.008$; Fig. $4 \mathrm{~A}$ and $\mathrm{B}$ ). CD13+ or CD33+/HLA-DR+ groups exhibited decreased OS ( 2 vs. 80 months; $\mathrm{P}=0.008)$ and PFS ( 2 vs. 31 months; 
A

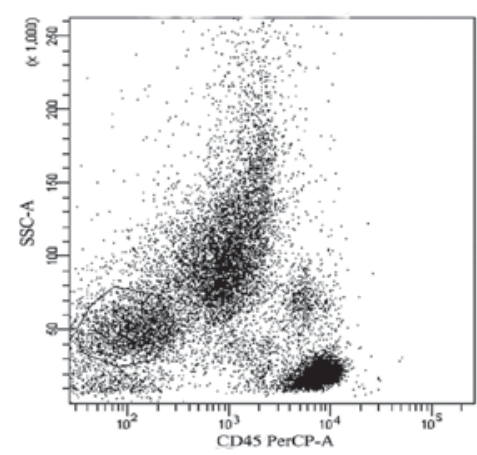

B

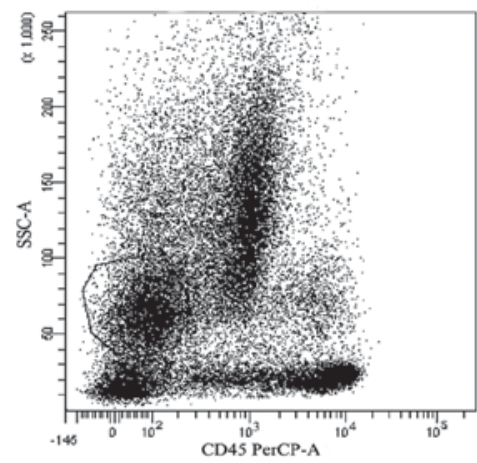

C

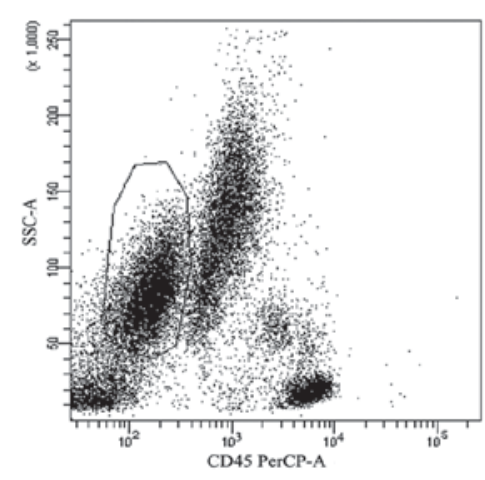

D

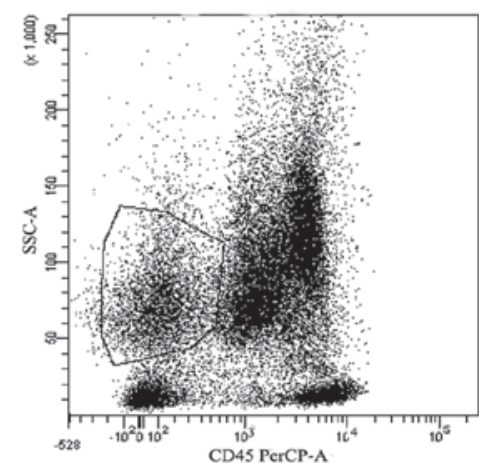

E

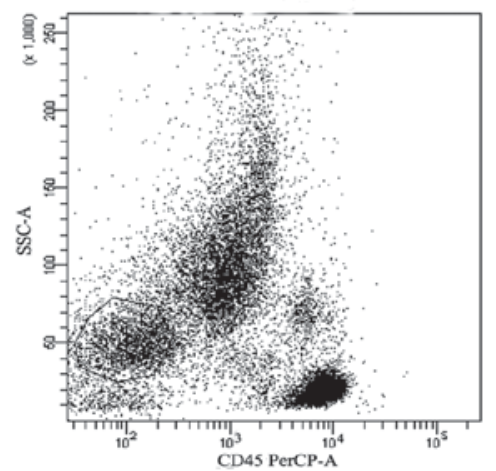

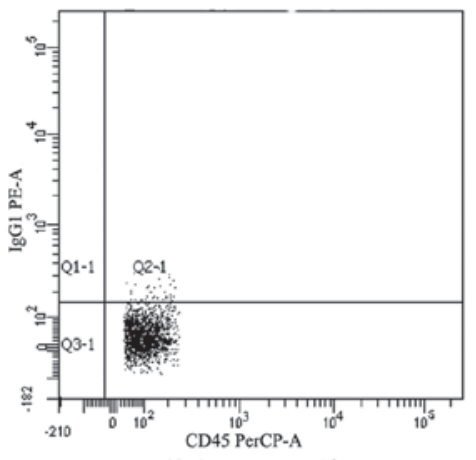
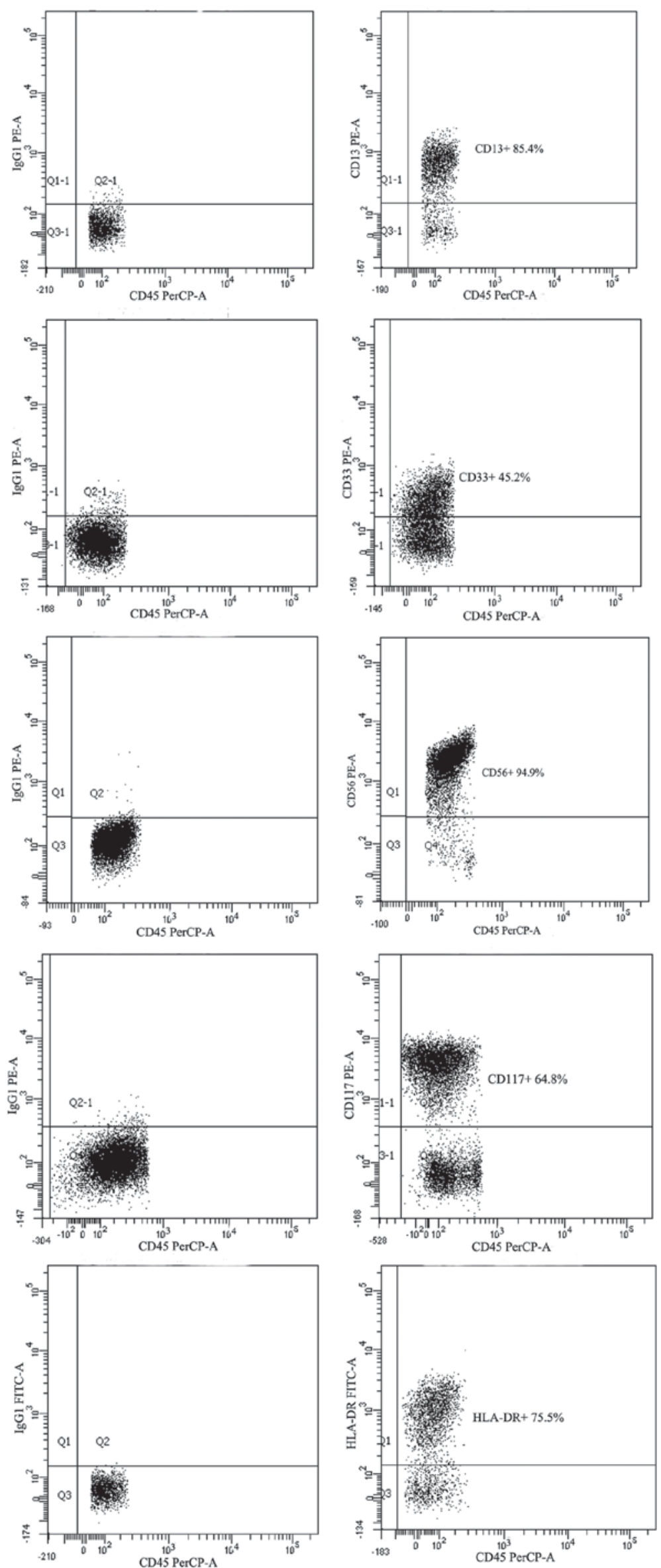

Figure 2. Flow cytometry data obtained from bone marrow samples. Expression of (A) CD13+, (B) CD33+, (C) CD56+, (D) CD117+ and (E) HLA-DR+. IgG1 was stained by (A-D) PE or (E) FITC as isotope/negative control. PE, phycoerythrin; FITC, fluorescein; PerCP, peridinin chlorophyll protein complex; HLA-DR, human leukocyte antigen-antigen D-related. 
Table III. Univariate analysis for overall survival and progression-free survival in patients with multiple myeloma.

A, Overall survival

\begin{tabular}{|c|c|c|c|c|}
\hline Characteristic & $\mathrm{B}$ & Wald & HR $(95 \% \mathrm{CI})$ & P-value \\
\hline Age, $\geq 65$ years & -0.617 & 3.984 & $1.853(1.011-3.394)$ & $0.046^{\mathrm{a}}$ \\
\hline Sex, male & -0.363 & 1.329 & $1.438(0.776-2.665)$ & 0.249 \\
\hline ISS stage, III & -0.725 & 5.417 & $2.066(1.121-3.805)$ & $0.020^{\mathrm{a}}$ \\
\hline Hemoglobin, $<100 \mathrm{~g} / \mathrm{l}$ & -0.548 & 2.267 & $1.729(0.848-3.527)$ & 0.132 \\
\hline Lymphocyte count, $>1.3 \times 10^{9} / 1$ & 0.519 & 2.646 & $1.681(0.899-3.141)$ & 0.104 \\
\hline C-reactive protein, $>8 \mathrm{mg} / \mathrm{l}$ & -0.645 & 4.071 & $0.525(0.281-0.982)$ & $0.044^{\mathrm{a}}$ \\
\hline Lactate dehydrogenase, $>245 \mathrm{U} / 1$ & -0.906 & 4.605 & $2.474(1.082-5.657)$ & $0.032^{\mathrm{a}}$ \\
\hline Creatinine, $>177 \mu \mathrm{mol} / 1$ & -0.316 & 0.813 & $1.372(0.690-2.726)$ & 0.367 \\
\hline $\mathrm{Ca}^{2+},>2.8 \mathrm{mmol} / 1$ & -0.289 & 0.483 & $1.335(0.591-3.017)$ & 0.487 \\
\hline Albumin, $<35 \mathrm{~g} / 1$ & -0.637 & 1.897 & $1.891(0.764-4.683)$ & 0.168 \\
\hline Chromosome, $\geq 3$ abnormal & -0.900 & 7.190 & $2.459(1.274-4.476)$ & $0.007^{\mathrm{a}}$ \\
\hline Hypertension, yes & -0.14 & 0.194 & $0.869(0.466-1.622)$ & 0.660 \\
\hline Diabetes, yes & -0.657 & 3.670 & $1.929(0.985-3.779)$ & 0.055 \\
\hline CD13, positive & -0.558 & 0.578 & $1.746(0.415-7.349)$ & 0.447 \\
\hline CD33, positive & -0.337 & 0.491 & $1.401(0.546-3.597)$ & 0.483 \\
\hline CD56, positive & -0.070 & 0.044 & $0.932(0.486-1.789)$ & 0.833 \\
\hline CD117, positive & -0.886 & 3.373 & $2.426(0.942-6.248)$ & 0.066 \\
\hline HLA-DR, positive & -1.143 & 8.18 & $3.135(1.433-6.860)$ & $0.004^{\mathrm{a}}$ \\
\hline
\end{tabular}

B, Progression-free survival

\begin{tabular}{|c|c|c|c|c|}
\hline Characteristic & $\mathrm{B}$ & Wald & $\mathrm{HR}(95 \% \mathrm{CI})$ & P-value \\
\hline Age, $\geq 65$ years & -0.418 & 1.931 & $1.519(0.842-2.738)$ & 0.165 \\
\hline Sex, male & -0.408 & 1.705 & $1.503(0.815-2.772)$ & 0.192 \\
\hline ISS stage, III & -0.633 & 3.824 & $1.883(0.999-3.552)$ & 0.051 \\
\hline Hemoglobin, $<100 \mathrm{~g} / \mathrm{l}$ & -0.341 & 0.927 & $1.407(0.702-2.817)$ & 0.336 \\
\hline Lymphocyte count, $>1.3 \times 10^{9} / 1$ & 0.238 & 0.606 & $1.268(0.697-2.308)$ & 0.436 \\
\hline C-reactive protein, $>8 \mathrm{mg} / \mathrm{l}$ & -0.633 & 3.933 & $1.882(1.007-3.517)$ & $0.047^{\mathrm{a}}$ \\
\hline Lactate dehydrogenase, $>245 \mathrm{U} / 1$ & -0.696 & 2.798 & $2.006(0.887-4.536)$ & 0.094 \\
\hline Creatinine, $>177 \mu \mathrm{mol} / 1$ & -0.250 & 0.498 & $1.284(0.641-2.571)$ & 0.480 \\
\hline $\mathrm{Ca}^{2+},>2.8 \mathrm{mmol} / \mathrm{l}$ & -0.569 & 1.867 & $1.766(0.781-3.995)$ & 0.172 \\
\hline Albumin, $<35 \mathrm{~g} / 1$ & -0.505 & 1.306 & $1.657(0.697-3.942)$ & 0.253 \\
\hline Chromosome, $\geq 3$ abnormal & -0.729 & 4.362 & $2.073(1.046-4.107)$ & $0.037^{\mathrm{a}}$ \\
\hline Hypertension, yes & -0.079 & 0.060 & $0.924(0.492-1.737)$ & 0.806 \\
\hline Diabetes, yes & -0.735 & 4.553 & $2.086(1.062-4.100)$ & $0.033^{\mathrm{a}}$ \\
\hline CD13, positive & -0.162 & 0.049 & $1.175(0.282-4.891)$ & 0.824 \\
\hline CD33, positive & -0.850 & 3.156 & $2.340(0.916-5.981)$ & 0.076 \\
\hline CD56, positive & 0.118 & 0.125 & $1.125(0.586-2.158)$ & 0.724 \\
\hline CD117, positive & -1.143 & 5.590 & $3.136(1.216-8.087)$ & $0.018^{\mathrm{a}}$ \\
\hline HLA-DR, positive & -0.999 & 6.389 & $2.715(1.251-5.892)$ & $0.011^{\mathrm{a}}$ \\
\hline
\end{tabular}

CI, confidence interval; ISS, international staging system; HLA-DR, human leukocyte antigen-antigen D-related. ${ }^{\mathrm{a}} \mathrm{P}<0.05$. B, regression coefficient; Wald, Wald Statistics; HR, hazard ratio.

$\mathrm{P}=0.03)$ compared with HLA-DR- immunophenotypes (Fig. 4C and D). A similar trend was observed for CD117+/HLA-DR+ compared with CD117+/HLA-DRimmunophenotypes for OS (2 vs. 33 months; $\mathrm{P}=0.018$;
Fig 4E). However, PFS did not exhibit a significant survival difference between CD117+/HLA-DR+ and CD117+/HLA-DR( 2 vs. 6 months; $\mathrm{P}=0.142$; Fig 4F). No significant differences were observed for OS and PFS between CD56+/HLA-DR+ 
Table IV. Multivariate analysis for overall survival and progression-free survival in patients with multiple myeloma.

A, Overall survival

\begin{tabular}{lcccc}
\hline Characteristic & B & Wald & HR (95\% CI) & P-value \\
\hline Age, $\geq 65$ years & -0.727 & 4.596 & $0.483(0.249-0.940)$ & $0.032^{\mathrm{a}}$ \\
ISS stage, III & -0.755 & 5.139 & $0.470(0.245-0.903)$ & $0.023^{\mathrm{a}}$ \\
C-reactive protein, $>8 \mathrm{mg} / \mathrm{l}$ & -0.525 & 2.309 & $0.529(0.301-1.164)$ & 0.129 \\
Lactate dehydrogenase, $>245 \mathrm{U} / 1$ & -0.763 & 2.975 & $0.466(0.196-1.110)$ & 0.085 \\
Chromosome, $\geq 3$ abnormal & -0.689 & 3.700 & $0.052(0.249-0.940)$ & 0.054 \\
HLA-DR, positive & -1.102 & 6.528 & $0.332(0.143-0.774)$ & $0.011^{\mathrm{a}}$ \\
\hline
\end{tabular}

B, Progression-free survival

\begin{tabular}{lcccc}
\hline Characteristic & B & Wald & HR (95\% CI) & P-value \\
\hline C-reactive protein, $>8 \mathrm{mg} / \mathrm{l}$ & -0.627 & 3.643 & $0.534(0.281-1.017)$ & 0.056 \\
Chromosome, $\geq 3$ abnormal & -0.558 & 2.108 & $0.572(0.269-1.216)$ & 0.146 \\
Diabetes, yes & -0.842 & 5.674 & $0.431(0.216-0.861)$ & $0.017^{\mathrm{a}}$ \\
CD117, positive & -1.082 & 4.639 & $0.339(0.127-0.902)$ & $0.030^{\mathrm{a}}$ \\
HLA-DR, positive & -0.902 & 4.238 & $0.404(0.170-0.957)$ & $0.040^{\mathrm{a}}$ \\
\hline
\end{tabular}

CI, confidence interval; ISS, international staging system; HLA-DR, human leukocyte antigen-antigen D-related; B, regression coefficient; Wald, Wald Statistics; HR, hazard ratio. ${ }^{\mathrm{a}} \mathrm{P}<0.05$.

Table V. Baseline characteristics for patients newly diagnosed with multiple myeloma and varying CD56/CD117 immunophenotypes.

\begin{tabular}{|c|c|c|c|c|}
\hline \multirow[b]{2}{*}{ Characteristic } & \multicolumn{3}{|c|}{ Number or median (range) } & \multirow[b]{2}{*}{ P-value } \\
\hline & CD56+/CD117+ & CD56+/CD117- or CD56-/CD117+ & CD56-/CD117- & \\
\hline $\mathrm{n}$ & 4 & 54 & 22 & \\
\hline Age, years & $68.5(43-75)$ & $61(24-80)$ & $62(47-75)$ & 0.62 \\
\hline Sex, male & 3 & 28 & 15 & 0.35 \\
\hline ISS stage III & 3 & 26 & 11 & 0.78 \\
\hline Hemoglobin, g/l & $85.50(79-94)$ & $88(45-131)$ & $83.50(59-148)$ & 0.89 \\
\hline Lymphocyte count, $x 10^{9} / 1$ & $2.34(0.72-1.42)$ & $1.20(0.16-3.45)$ & $1.24(0.62-3.95)$ & 0.79 \\
\hline C-reactive protein, $\mathrm{mg} / \mathrm{l}$ & $17(2-49)$ & $5(1-77)$ & $5(1-160)$ & 0.59 \\
\hline Lactate dehydrogenase, U/l & $176(109-315)$ & $127(61-1,221)$ & $133(50-365)$ & 0.23 \\
\hline Creatinine, $\mu \mathrm{mol} / 1$ & $88.35(55.20-117)$ & $97.50(43.30-760.90)$ & $100.55(30.30-372.70)$ & 0.65 \\
\hline $\mathrm{Ca}^{2+}, \mathrm{mmol} / \mathrm{l}$ & $2.20(1.99-2.31)$ & $2.20(1.38-5.35)$ & $2.20(1.93-2.95)$ & 0.78 \\
\hline Albumin, $\mathrm{g} / \mathrm{l}$ & $29.65(17.10-34.10)$ & $27.70(11.70-44.10)$ & $29.20(20.10-42.70)$ & 0.81 \\
\hline$\beta-2$ microglobulin, $\mathrm{mg} / \mathrm{l}$ & $7.30(5.40-26.80)$ & $5.35(1.50-81.88)$ & $5.45(1.88-39.02)$ & 0.38 \\
\hline Plasma cells in bone marrow, $\%$ & $17.25(10.50-75)$ & $27.75(10-95)$ & $35.50(12-86)$ & 0.77 \\
\hline Chromosome, $\geq 3$ abnormal & 1 & 10 & 3 & 0.78 \\
\hline Hypertension, yes & 1 & 20 & 9 & 0.93 \\
\hline Diabetes, yes & 1 & 13 & 3 & 0.49 \\
\hline
\end{tabular}

and CD56+/HLA-DR- immunophenotypes $(\mathrm{P}=0.194$ and $\mathrm{P}=0.189$; respectively; Fig $4 \mathrm{G}$ and $\mathrm{H})$.

Derivation of myeloma prognostic index. The myeloma prognostic index (MPI) was derived from the variables that were determined to be significant prognostic factors for OS based on the multivariate analysis. HLA-DR+, age $\geq 65$ years and ISS stage III exhibited an independent, unfavorable significance for OS. Here, the MPI attributed 1 point for HLA-DR+, old age ( $\geq 65$ years) and ISS (stage III) and the final score was obtained. Risk categories were stratified into low (score, 0 ), intermediate (score, 1) and high (score, 2-3). The low, 
Table VI. Baseline characteristics for patients newly diagnosed with multiple myeloma and varying HLA-DR immunophenotypes.

Number or median (range)

\begin{tabular}{|c|c|c|c|}
\hline Characteristic & HLA-DR- & HLA-DR+ & P-value \\
\hline $\mathrm{n}$ & 72 & 8 & \\
\hline Age, years & $62.50(56-75)$ & $61(24-80)$ & 0.34 \\
\hline Sex, male & 39 & 7 & 0.15 \\
\hline ISS stage III & 38 & 2 & 0.26 \\
\hline Hemoglobin, g/l & $81.50(64-112)$ & $88(45-148)$ & 0.30 \\
\hline Lymphocyte count, $\mathrm{x} 10^{9} / 1$ & $1.19(0.53-3.00)$ & $1.20(0.16-3.95)$ & 0.89 \\
\hline C-reactive protein, mg/l & $10(1-51)$ & $5(1-160)$ & 0.44 \\
\hline Lactate dehydrogenase, U/l & $158(69-1,221)$ & $127(50-1,102)$ & 0.49 \\
\hline Creatinine, $\mu \mathrm{mol} / 1$ & $88.95(57.2-341.7)$ & $100.50(30.30-760.90)$ & 0.59 \\
\hline $\mathrm{Ca}^{2+}, \mathrm{mmol} / \mathrm{l}$ & $2.06(1.93-3.25)$ & $2.20(1.38-5.35)$ & 0.28 \\
\hline Albumin, $g / 1$ & $28.90(17.70-34.10)$ & $28.20(11.70-44.10)$ & 0.46 \\
\hline$\beta-2$ microglobulin, $\mathrm{mg} / \mathrm{l}$ & $4.10(1.88-8.29)$ & $5.63(1.50-81.88)$ & 0.13 \\
\hline Plasma cells in bone marrow, $\%$ & $38(11.50-95)$ & $26.50(10.50-92)$ & 0.46 \\
\hline Chromosome, $\geq 3$ abnormal & 9 & 5 & $0.002^{\mathrm{a}}$ \\
\hline Hypertension, yes & 28 & 2 & 0.70 \\
\hline Diabetes, yes & 16 & 1 & 0.86 \\
\hline
\end{tabular}

${ }^{\mathrm{a}} \mathrm{P}<0.05$.
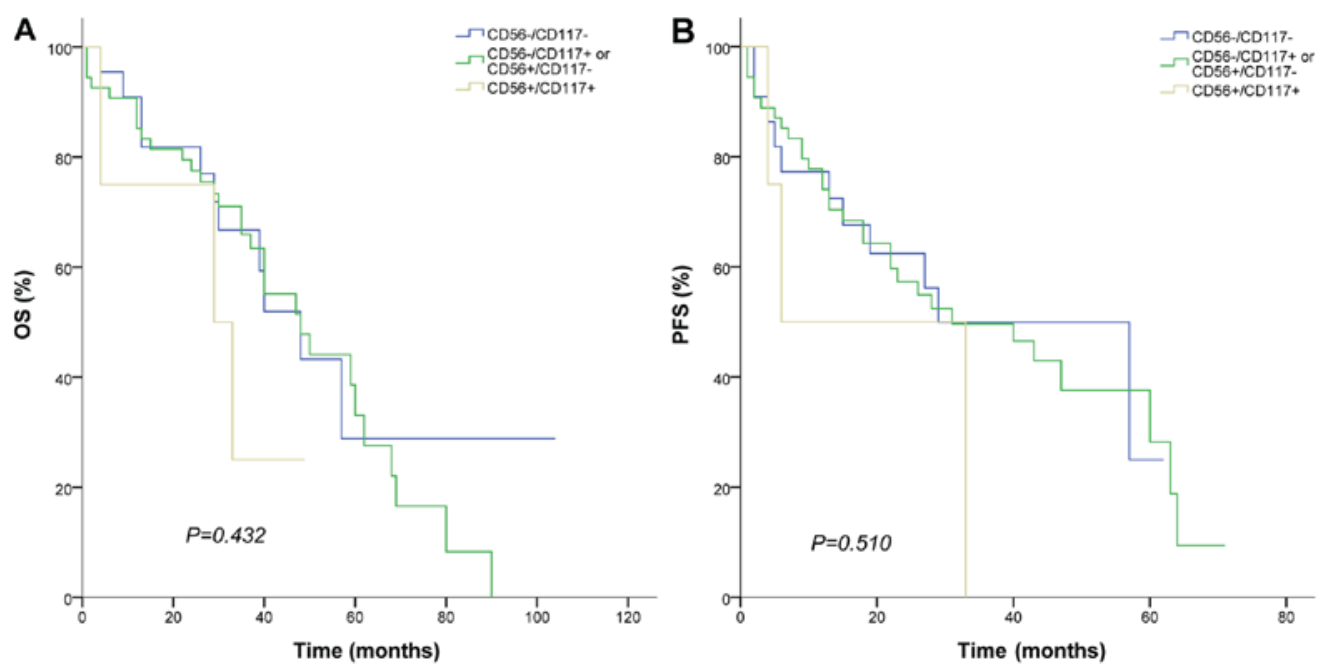

Figure 3. Kaplan-Meier analysis of (A) OS and (B) PFS in patients grouped as CD56+/CD117+, CD56-/CD117- and CD56+/CD117- or CD56-/CD117+. OS, overall survival; PFS, progression-free survival.

intermediate and high MPI was suitable for risk stratification in patients treated with bortezomib and thalidomide for OS (60, 47 and 33 months, respectively; $\mathrm{P}<0.001)$ and PFS (60, 28 and 12 months, respectively; $\mathrm{P}=0.004$; Fig. 5). Low group and intermediate group had significant differences in OS (60 vs. 47 months; $\mathrm{P}=0.011$ ) and PFS (60 vs. 28 months; $\mathrm{P}=0.038)$. Intermediate group had longer $\mathrm{OS}$ than high group (47 vs. 33 months; $\mathrm{P}=0.013$ ) but their PFS did not show the same difference ( 28 vs. 12 months; $\mathrm{P}=0.076$ ). In addition, patients with complex chromosome had a shorter OS (13 vs. 57 months; $\mathrm{P}=0.005)$ and PFS (9 vs. 47 months; $\mathrm{P}=0.032$ ) (Fig 6).

\section{Discussion}

As a currently incurable disease, research focusing on MM is ongoing. Developments in the fields of biotechnology and the identification of novel drugs have furthered the understanding of the disease and aided the improvement of treatments (1). It has been demonstrated that MM in different R-ISS stages consists of interphase fluorescence in situ hybridization (FISH), ß2-microglobulin, lactate dehydrogenase and albumin, have different prognosis, R-ISS consists of interphase fluorescence in situ hybridization (FISH), $\beta 2$-microglobulin, lactate 

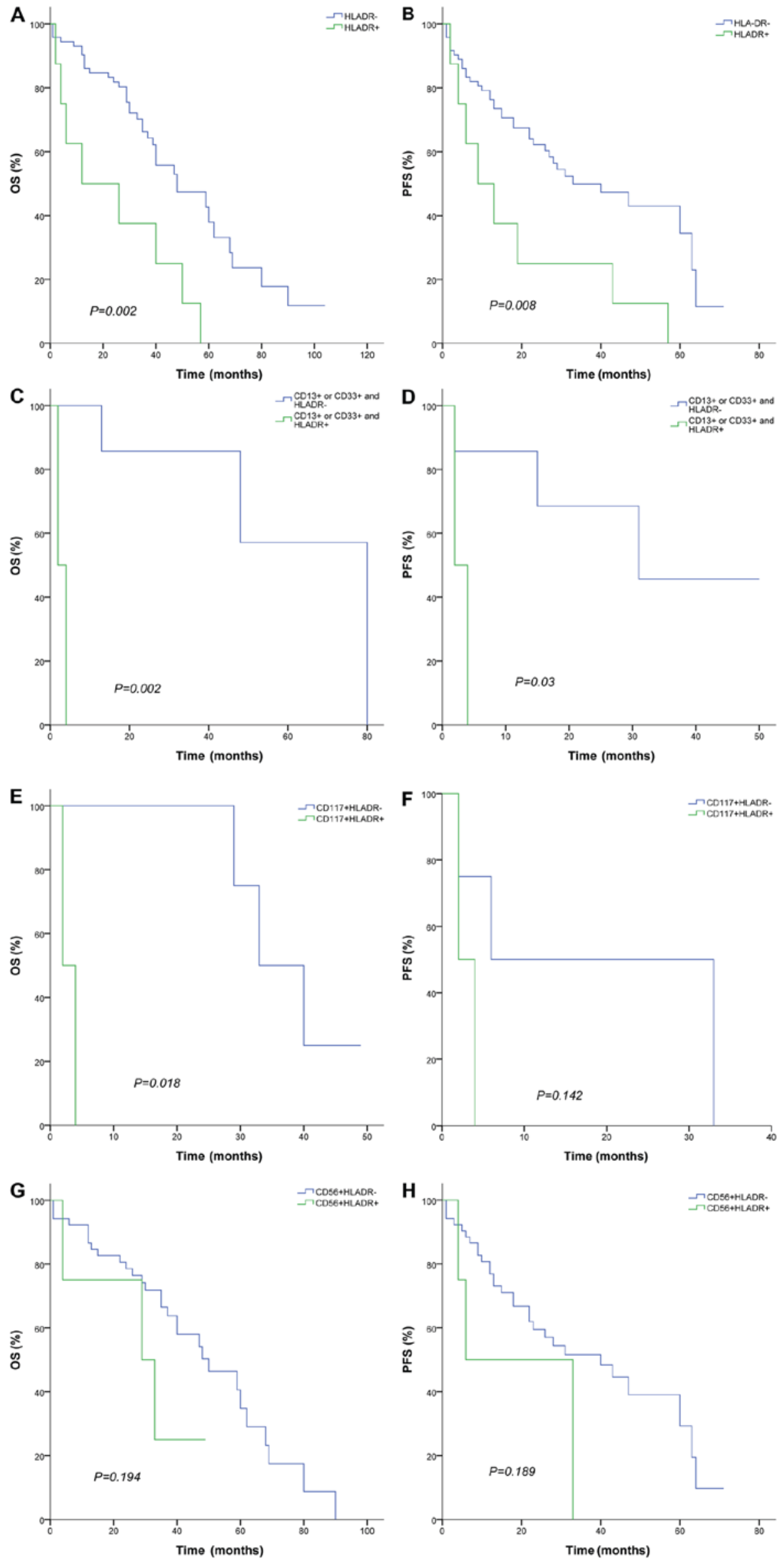

Figure 4. Kaplan-Meier analysis of OS and PFS in varying immunophenotypes. (A) OS and (B) PFS associated with HLA-DR expression. (C) OS and (D) PFS associated with CD13+ or CD33+ and HLA-DR expression. (E) OS and (F) PFS associated with CD117+ and HLA-DR expression. (G) OS and (H) PFS associated with CD56+ and HLA-DR expression. OS, overall survival; PFS, progression-free survival; HLA-DR, human leukocyte antigen-antigen D-related. 

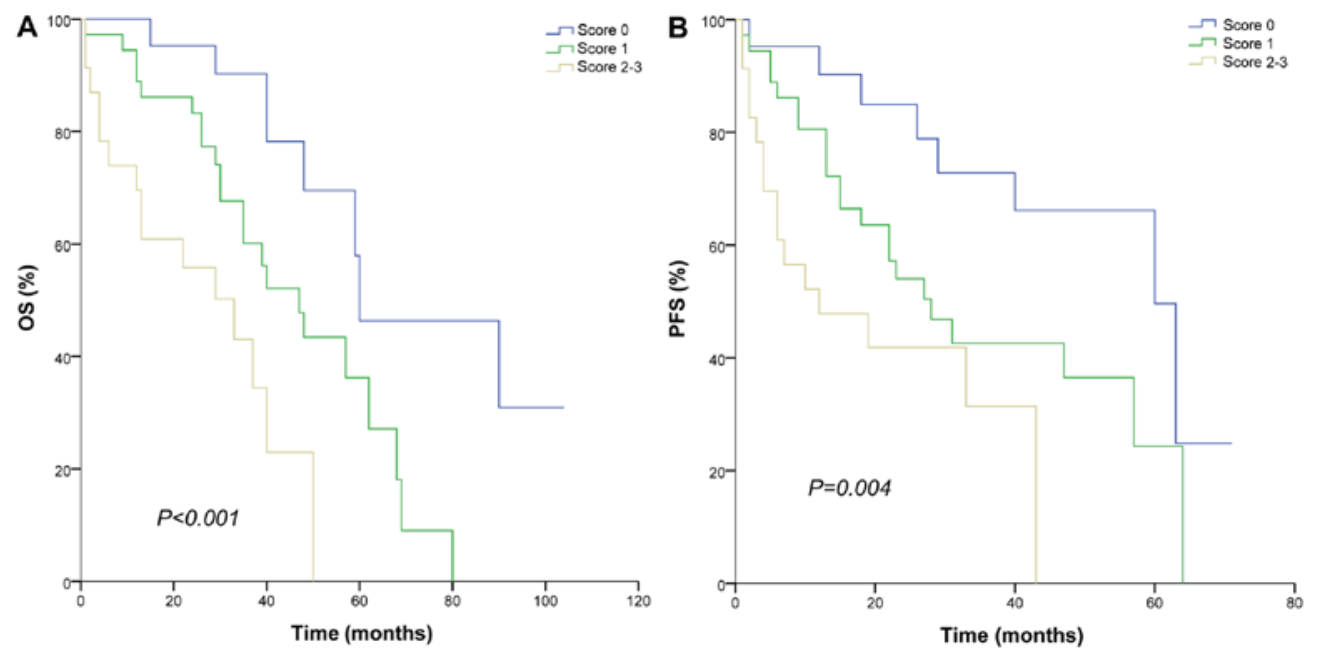

Figure 5. Survival curve according to MPI categories. (A) OS and (B) PSF for patients of different risk categories. MPI attributed 1 point for HLA-DR+, age, $\geq 65$ years and international staging system stage III and risk categories were stratified into low (score, 0), intermediate (score, 1) and high (score, 2-3). MPI, myeloma prognostic index; OS, overall survival; PFS, progression-free survival; HLA-DR, human leukocyte antigen-antigen D-related.
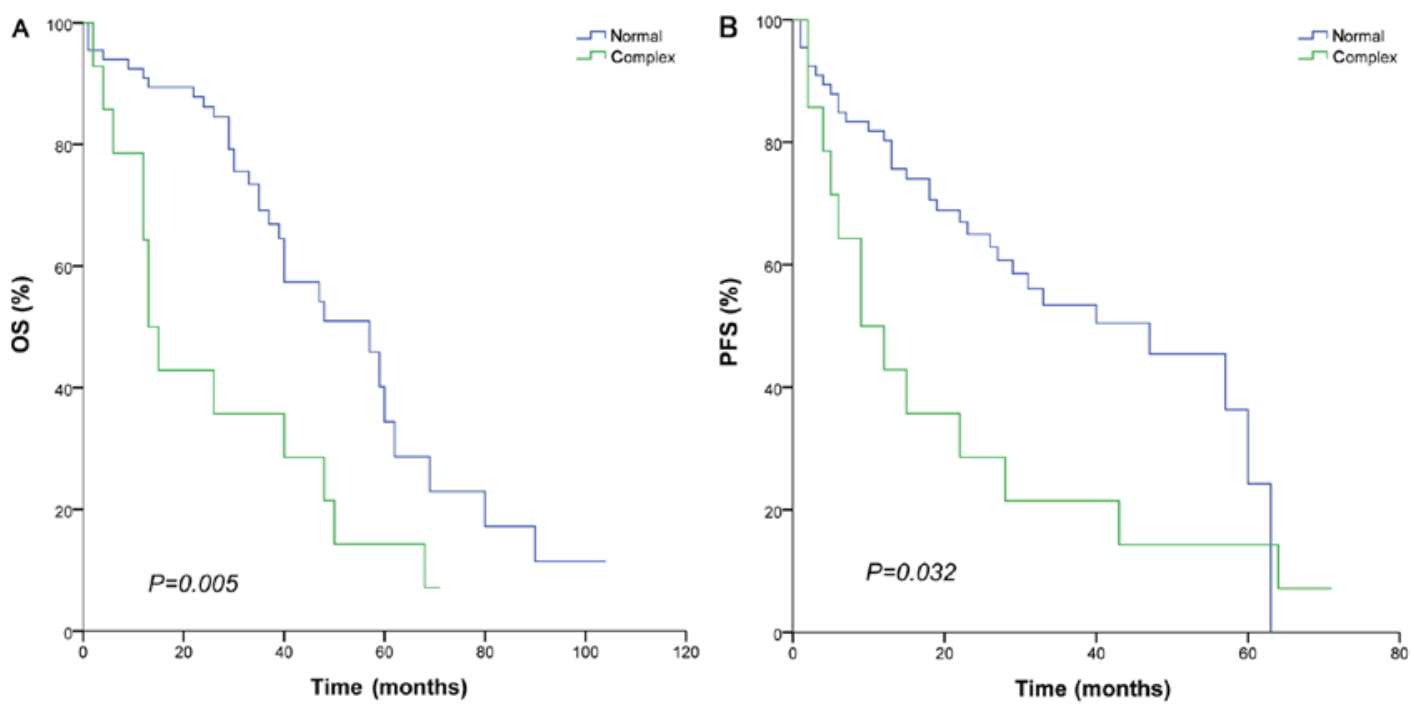

Figure 6. Kaplan-Meier analysis of OS and PFS associated with complex chromosomes. (A) OS and (B) PFS for patients with multiple myeloma with and without complex chromosomes. OS, overall survival; PFS, progression-free survival; complex, $\geq 3$ abnormal chromosomes.

dehydrogenase and albumin. Patients in different stages had a different prognosis (15). Treatment with lenalidomide provides an alternative choice for elderly patients and those unable to tolerate chemotherapy $(16,17)$. In addition, prognostic factors, including peripheral lymphocytes and CRP, have been reported and were demonstrated to have a varying prognostic significance in traditional chemotherapy and novel drug protocols $(4,5,18,19)$. This highlights the importance of discovering and identifying prognostic markers in MM for novel treatment approaches.

Immunophenotyping, as a critical cell indicator, is a necessary step in the diagnosis of MM (6). It is known that MM plasma cells express CD138 and CD38 at high levels, are positive for CD56, partially positive for CD117 and do not express CD27. Normal bone marrow plasma cells do not express CD138 and CD38. They are CD56 negative, express CD19 weakly compared with MM plasma cells and express CD45 $(6,7,20)$. In the current study, all patients expressed CD38 and $70.00 \%$
(56 cases) were CD56 and $13.75 \%$ (11 cases) were CD117 positive, which is in accordance with previous reports $(7,8)$.

In addition to the exact diagnostic significance, the prognostic value of immunophenotyping has been documented for MM (21-24). Previously, Dahl et al (21) reported that the absence of CD56 may contribute to extramedullar accumulation of tumor cells and Bataille et al (22) reported CD117+ patients exhibited an increased OS compared with negative individuals. In addition, it was reported that the 3-year OS in CD33+ patients was significantly decreased compared with CD33- patients (23). Recently, Pan et al (8) suggested that CD56 and CD117 expression has a potential prognostic impact and it may be associated with increased OS. Ceran et al (9) reported that CD56 and CD117 expression levels are lower in advanced stages of MM. Qiu et al (24) reported that OS and PFS in CD56+ patients with MM are increased compared with CD56- patients treated with lenalidomide or bortezomib-based therapies. It was suggested that CD56 and CD117 negative 
expression are unfavorable prognostic factors in patients with MM, based on data obtained from small cohorts (34-50 participants), non-combination therapy and uniformed protocols i.e., using novel agents and traditional chemotherapies $(8,9)$.

Opposing trends have also been reported. According to data from Tang et al (25), therapeutic efficacy of CD117+ patients was decreased compared with CD117- patients. Mateo et al (26) documented that patients with MM and CD117+ treated with conventional therapy, including therapy followed by autologous stem-cell transplantation, had decreased OS and PFS compared with CD117- patients with MM. The prognostic judgment based on immunophenotype indicators remains controversial. According to the current study, no significant difference was observed in OS and PFS between CD56+/CD117+ and CD56-/CD117- patients and these markers did not appear to be associated with poor prognosis. Differences in the described outcomes may be attributed to the following: Previous reports $(8,9,24)$ resulted from monotherapy, including iMiDs, proteasome inhibitors or traditional chemotherapy, or intermittent iMiDs administration and these results were based on combination therapy, including thalidomide which were given continuously in the presence or absence of chemotherapy; a majority of patients tolerated small doses of thalidomide and peripheral neuritis was accepted. A uniform protocol was also applied. Elevation in CD117 and CD56 levels reported by Pan et al (8) and Ceran et al (9) resulted from varying treatments, including chemotherapies and novel agents. The influence of different regimes and potential resulting bias was not accounted for. Hundemer et al (27) reported that a lack of CD56 expression on MM cells was not an indicator for poor prognosis in patients with MM treated with a high chemotherapy dose followed by autologous hematopoietic stem cell transplantation. The present study suggested that CD117+ remained a negative factor in patients with MM treated with novel agents. However, larger cohort studies are required to confirm these conclusions.

According to univariate analysis, HLA-DR+ expression was associated with decreased OS and PFS and similar results were observed in combination with CD117+ or myeloid antigen (CD13+ or CD33+) expression. In addition, HLA-DR+ expression was identified as an independent factor for decreased OS and PFS. To the best of the our knowledge, the current study is the first to report effects of HLA-DR expression on OS and PFS in patients with MM.

The HLA gene, located on the short arm of chromosome 6 , is a $4,000 \mathrm{~kb}$ gene complex composed of various tightly connected gene clusters, which are known for maximum allele polymorphisms and are closely associated with functions of the immune system in humans (28). The HLA-DR antigen, which is encoded by the HLA-II gene, most frequently appears on the cytomembrane of macrophages and B lymphocytes and may aid the host immune system to identify and attack tumor cell (28). Previously, research regarding HLA-DR concentrated on diseases associated with the immune system $(29,30)$ and solid tumors. Diao et al (31) indicated that overexpression of HLA-DR is associated with decreased OS in patients with glioma, while Sconocchia et al (32) reported that HLA class II antigen served as a favorable prognostic marker in patients with colorectal carcinoma. In hematological malignances, certain reports have suggested that the HLA-DR status determined by flow cytometry may be used to predict the prognosis of patients with diffuse large $\mathrm{B}$-cell lymphoma receiving $\mathrm{R}-\mathrm{CHOP}$ (rituximab, cyclophosphamide, doxorubicin, vincristine, prednisone) therapy $(33,34)$. The current study demonstrated that HLA-DR positive is an unfavorable factor for OS and PFS in patients with MM. HLA-DR+ may be a valuable and simple prognostic marker in the field of novel therapies. However, larger studies with increased follow-up and additional researches are required in the future to confirm the presented conclusions, particularly in patients with transplantations. Co-expression with CD117 or myeloid antigens (CD13 or CD33) did not alter the exhibited influence. This may result from a close association with complex chromosomes. Although $\mathrm{t}(4 ; 14), \mathrm{t}(14 ; 16)$ and $17 \mathrm{p}$ - were previously reported as unfavorable karyotypes in revised ISS for MM (R-ISS), further unknown abnormalities may have been omitted as a result of restrictions of the FISH probes (15). Certain complex karyotypes detected were based on traditional reverse-banding and/or G-banding techniques. According to the current study, complex chromosomes may be a valuable prognostic factor, while complex karyotypes have previously been described as an adverse prognostic factor (35). To the best of our knowledge the association between complex chromosomes and immunophenotypes has not been reported previously. However, these findings may be only be applicable for a Chinese population.

As a first generation immunoregulator, thalidomide is more widely used in China compared with lenalidomide, as a result of its lower price and a majority of patients with MM insisting on a combination therapy based on thalidomide and bortezomib (36). Flow cytometry, as an established technique, has been widely used in China (7). In association with simple ISS, R-ISS is not common in basic hospitals, and age or MPI may provide a simple diagnostic to predict prognosis in patients not undergoing transplantation. The use of immunophenotypes as prognostic markers is a simpler method than R-ISS. The current study suggested that the expression of CD117 and HLA-DR may be prognostic markers for novel therapies where combination therapy with thalidomide and bortezomib is predominant, however, further associations for the combination with transplantation should be studied.

There are several limitations associated with the current study. Certain international prognostic factors, including free light chain levels (FLC) were excluded as total light chain levels not FLC were measured until 2012. Patient numbers in the current study were increased compared with previous reports, however, following subdivision of the cohort, individual groups remained small $(8,9)$. Future perspectives include the recruitment of more patients and the analysis of more factors, including FLC, to identify further markers of long-term prognosis in patients with MM.

\section{Acknowledgements}

The authors would like to thank Mrs Ying Yin and Mrs Zheng You for assistance with data collection.

\section{Funding}

No funding was received. 


\section{Availability of data and materials}

The datasets used and/or analyzed during the current study are available from the corresponding author on reasonable request.

\section{Authors' contributions}

JNY, JWZ and HFG provided the raw data. HW and XZ analyzed and interpreted the patient data and drafted the manuscript. CS designed the study and revised the manuscript. All authors read and approved the final manuscript.

\section{Ethics approval and consent to participate}

This retrospective study was approved by the Institutional Review Board of Wuxi People's Hospital (Wuxi, China) in accordance with the Declaration of Helsinki and informed consent to participate in the study was obtained from participants.

\section{Patient consent for publication}

Not applicable.

\section{Competing interests}

The authors declare that they have no competing interests.

\section{References}

1. Cejalvo MJ and de la Rubia J: Which therapies will move to the front line for multiple myeloma? Expert Rev Hematol 10: 383-392, 2017.

2. Palumbo A and Anderson K: Multiple myeloma. N Engl J Med 364: 1046-1060, 2011.

3. Anderson KC, Alsina M, Bensinger W, Biermann JS, Cohen AD, Devine S, Djulbegovic B, Faber EA Jr, Gasparetto C, Hernande Z-Illizaliturri F, et al: Multiple myeloma, version 1.2013. J Natl Compr Canc Netw 11:11-17, 2013.

4. Tadmor T: Do lymphocytes count in myeloma? Are we absolutely sure? Leuk Lymphoma 56: 1193-1194, 2015.

5. Kim DS, Yu ES, Kang KW, Lee SR, Park Y, Sung HJ, Choi CW and Kim BS: Myeloma prognostic index at diagnosis might be a prognostic marker in patients newly diagnosed with multiple myeloma. Korean J Intern Med 32: 711-721, 2017.

6. Gertz MA and Buadi FK: Utility of immunophenotyping of plasma cells in multiple myeloma. Leuk Lymphoma 28: 1-2, 2015.

7. Li HQ and Zhai YP: Research progress on multiple myeloma immunophenotyping and minimal residual disease detected by flow cytometry. Zhongguo Shi Yan Xue Ye Xue Za Zhi 23: 241-245, 2015 (In Chinese).

8. Pan Y, Wang H, Tao Q, Zhang C, Yang D, Qin H, Xiong S, Tao L, Wu F, Zhang J and Zhai Z: Absence of both CD56 and CD117 expression on malignant plasma cells is related with a poor prognosis in patients with newly diagnosed multiple myeloma. Leuk Res 40: 77-82, 2016.

9. Ceran F, Falay M, Dagdas S, and Ozet G: The assessment of CD56 and CD117 expressions at the time of the diagnosis in multiple myeloma patients. Turk J Haematol 34: 226-232, 2017.

10. International Myeloma Working Group: Criteria for the classification of monoclonal gammopathies, multiple myeloma and related disorders: A report of the international myeloma working group. Br J Haematol 121: 749-757, 2003.

11. Greipp PR, San Miguel J, Durie BG, Crowley JJ, Barlogie B Blade J, Boccadoro M, Child JA, Avet-Loiseau H, Kyle RA, et al: International staging system for multiple myeloma. J Clin Oncol 23: 3412-3420, 2005.

12. Cavo M, Rajkumar SV, Palumbo A, Moreau P, Orlowski R, Blade J, Sezer O, Ludwig H, Dimopoulos MA, Attal M, et al International Myeloma Working Group consensus approach to the treatment of multiple myeloma patients who are candidates for autologous stem cell transplantation. Blood 117: 6063-6073, 2011.
13. Palumbo A,Rajkumar SV, San Miguel JF,Larocca A, Niesvizky R, Morgan G, Landgren O, Hajek R, Einsele H, Anderson KC, et al: International Myeloma Working Group consensus statement for the management, treatment, and supportive care of patients with myeloma not eligible for standard autologous stem-cell transplantation. J Clin Oncol 32: 587-600, 2014.

14. Durie BG, Harousseau JL, Miguel JS, Blade J, Barlogie B, Anderson K, Gertz M, Dimopoulos M, Westin J, Sonneveld $\mathrm{P}$, et al: International uniform response criteria for multiple myeloma. Leukemia 20: 1467-1473, 2006

15. Palumbo A, Avet-Loiseau H, Oliva S, Lokhorst HM, Goldschmidt H, Rosinol L, Richardson P, Caltagirone S, Lahuerta JJ, Facon T, et al: Revised international staging system for multiple myeloma: A Report From International Myeloma Working Group. J Clin Oncol 33: 2863-2869, 2015.

16. Benboubker L, Dimopoulos MA, Dispenzieri A, Catalano J, Belch AR, Cavo M, Pinto A, Weisel K, Ludwig H, Bahlis N, et al: Lenalidomide and dexamethasone in transplant-ineligible patients with myeloma. N Engl J Med 371: 906-917, 2014.

17. Gay F, Oliva S, Petrucci MT, Conticello C, Catalano L, Corradini P, Siniscalchi A, Magarotto V, Pour L, Carella A, et al: Chemotherapy plus lenalidomide versus autologous transplantation, followed by lenalidomide plus prednisone versus lenalidomide maintenance, in patients with multiple myeloma: A randomised, multicentre, phase 3 trial. Lancet Oncol 16: $1617-1629,2015$.

18. Jimenez-Zepeda VH, Reece DE, Trudel S, Chen C, Franke N, Winter A, Tiedemann R and Kukreti V: Absolute lymphocyte count as predictor of overall survival for patients with multiple myeloma treated with single autologous stem cell transplant. Leuk Lymphoma 56: 2668-2673, 2015.

19. Suriu C, Akria L, Azoulay D, Shaoul E, Barhoum M and Braester A: Absolute lymphocyte count as a prognostic marker in newly diagnosed multiple myeloma patients. Int J Lab Hematol 38: e56-59, 2016.

20. Bataille R, Jego G, Robillard N, Barille-Nion S, Harousseau JL, Moreau P, Amiot M and Pellat-Deceunynck C: The phenotype of normal, reactive and malignant plasma cells. Identification of 'many and multiple myelomas' and of new targets for myeloma therapy. Haematologica 91: 1234-1240, 2006.

21. Dahl IM, Rasmussen T, Kauric G and Husebekk A: Differential expression of CD56 and CD44 in the evolution of extramedullary myeloma. Br J Haematol 116: 273-277, 2002.

22. Bataille R, Pellat-Deceunynck C, Robillard N, Avet-Loiseau H, Harousseau JL and Moreau P: CD117 (c-kit) is aberrantly expressed in a subset of MGUS and multiple myeloma with unexpectedly good prognosis. Leuk Res 32: 379-382, 2008.

23. Sahara N, Ohnishi K, Ono T, Sugimoto Y, Kobayashi M, Takeshita K, Shigeno K, Nakamura S, Naito K, Tamashima S, et al: Clinicopathological and prognostic characteristics of CD33-positive multiple myeloma. Eur J Haematol 77: 14-18, 2006.

24. Qiu Q, Zhu P, Wang MJ, Lu XZ, Dong YJ, Sun YH, Wang LH, Zhang Y, Bu DF, Wang WS, et al: Expression of CD56 and CD19 in patients with newly diagnosed multiple myeloma and their relationship with karyotypes and prognosis. Zhongguo Shi Yan Xue Ye Xue Za Zhi 24: 1071-1078, 2016 (In Chinese).

25. Tang HL, Shu MM, Dong BX, Gu HT, Liang R, Bai QX, Yang L, Zhang T, Gao GX and Chen XQ: Influence of CD117 expression on response of multiple myeloma patients to chemotherapy. Zhongguo Shi Yan Xue Ye Xue Za Zhi 23: 1346-1351, 2015 (In Chinese).

26. Mateo G, Montalban MA, Vidriales MB, Lahuerta JJ, Mateos MV Gutierrez N, Rosinol L, Montejano L, Blade J, Martinez R, et al: Prognostic value of immunophenotyping in multiple myeloma: A study by the PETHEMA/GEM cooperative study groups on patients uniformly treated with high-dose therapy. J Clin Oncol 26: 2737-2744, 2008.

27. Hundemer M, Klein U, Hose D, Raab MS, Cremer FW, Jauch A, Benner A, Heiss C, Moos M, Ho AD and Goldschmidt H: Lack of CD56 expression on myeloma cells is not a marker for poor prognosis in patients treated by high-dose chemotherapy and is associated with translocation $\mathrm{t}(11 ; 14)$. Bone Marrow Transplant 40: 1033-1037, 2007.

28. Complete sequence and gene map of a human major histocompatibility complex. The MHC sequencing consortium. Nature 401: 921-923, 1999.

29. Hasan ZN, Zalzala HH, Mohammedsalih HR, Mahdi BM, Abid LA, Shakir ZN and Fadhel MJ: Association between human leukocyteantigen-DR and demylinating Guillain-Barre syndrome. Neurosciences 19: 301-305, 2014. 
30. Nada AM and Hammouda M: Immunoregulatory T cells, LFA-3 and HLA-DR in autoimmune thyroid diseases. Indian J Endocrinol Metab 18: 574-581, 2014.

31. Diao J, Xia T, Zhao H, Liu J, Li B and Zhang Z: Overexpression of HLA-DR is associated with prognosis of glioma patients. Int J Clin Exp Pathol 8: 5485-5490,2015.

32. Sconocchia G, Eppenberger-Castori S, Zlobec I, Karamitopoulou E, Arriga R, Coppola A, Caratelli S, Spagnoli GC, Lauro D, Lugli A, et al: HLA class II antigen expression in colorectal carcinoma tumors as a favorable prognostic marker. Neoplasia 16: 31-42, 2014.

33. Rimsza LM, Leblanc ML, Unger JM, Miller TP, Grogan TM, Persky DO, Martel RR, Sabalos CM, Seligmann B, Braziel RM, et al: Gene expression predicts overall survival in paraffin-embedded tissues of diffuse large B-cell lymphoma treated with R-CHOP. Blood 112: 3425-3433, 2008.

34. Yamamoto W, Nakamura N, Tomita N, Takeuchi K, Ishii Y, Takahashi H, Watanabe R, Takasaki H, Motomura S, Kobayashi S, et al: Human leukocyte antigen-DR expression on flow cytometry and tumor-associated macrophages in diffuse large B-cell lymphoma treated by rituximab, cyclophosphamide, doxorubicin, vincristine and prednisone therapy: Retrospective cohort study. Leuk Lymphoma 55: 2721-2727, 2014.
35. Nemec P, Zemanova Z, Kuglik P, Michalova K, Tajtlova J, Kaisarova P, Oltova A,Filkova H, Holzerova M,Balcarkova J, et al: Complex karyotype and translocation $\mathrm{t}(4 ; 14)$ define patients with high-risk newly diagnosed multiple myeloma: Results of CMG2002 trial. Leuk Lymphoma 53: 920-927, 2012.

36. Chen SY: Clinical effect of bortezomib combined with dexamethasone and thalidomide on treatment of multiple myeloma, J Clin Med Practice 18: 124-126, 2014.

This work is licensed under a Creative Commons Attribution-NonCommercial-NoDerivatives 4.0 International (CC BY-NC-ND 4.0) License. 